\title{
Auditoria Contínua: Tendências No Cenário Brasileiro
}

\author{
Continuous Auditing: Scenario Trends In Brazil
}

\author{
Claudia Marchioti Nicolau dos Reis \\ claudia_marchioti@yahoo.com.br \\ UERJ
}

\author{
Fernando Pereira Tostes \\ fernando.tostes@uol.com.br \\ UERJ
}

Andréa Paula Osório Duque

andreapauladuque@gmail.com

UERJ

\begin{abstract}
Resumo: O presente estudo objetiva investigar as tendências de pesquisas acadêmicas predominantes no Brasil, na área de Auditoria Contínua, no período de 2006 a 2011, considerando os periódicos brasileiros Qualis Capes A2, B1, B2 e B3. A relevância do estudo consiste em colaborar na identificação da evolução quantitativa e qualitativa da pesquisa em Auditoria Contínua no Brasil. A metodologia utilizada baseou-se em um estudo exploratório e abordagem quanti-qualitativa. Os resultados obtidos contemplando a temática pesquisada apontam que apenas três artigos em periódicos nacionais abordam o assunto Auditoria Contínua, possivelmente justificada pelo desconhecimento do tema ou desinteresse por parte dos auditores e acadêmicos. No entanto, as pesquisas indicam a Auditoria Contínua como tendência de gestão e controle nos negócios devido ao aumento da complexidade dos dados e a preparação para atender aos órgãos reguladores.
\end{abstract}

Palavras-chave: Auditoria. Auditoria Contínua. Auditoria Digital. Relatórios Financeiros On-line.

Abstract: This study investigates the trends that predominate in Brazil in the field of Continuous Auditing, during the period from 2006 to 2011. It includes the Qualis CAPES Brazilian publicacions $\mathrm{A} 2, \mathrm{~B} 1, \mathrm{~B} 2$ and $\mathrm{B} 3$. The relevance of this study is to identify the volume of quantitative and qualitative research in Continuous Auditing in Brazil. The methodology used was based on exploratory and qualitative-quantitative approach. The results obtained showed that only three papers in Brazilian journals covered the subject, possibly explained by ignorance of the subject or lack of interest on the part of Brazilian auditors and academics. However, the study results indicate a trend in the application of Continuous Auditing and management control in business due to the increased complexity of business environment, data volume and time to prepare reports to meet the ever increasing demand from regulations.

Keywords: Auditing. Continuous Auditing. Digital Auditing. On-line Financial Reports.

Artigo recebido em: 01.11.2012; Aceito em: 30.01.2013

\section{INTRODUÇÃ̃O}


Os avanços tecnológicos de suporte à informação vêm contribuindo para a dispersão do conhecimento em ambiente Web, fatos que têm pontuado, também, a divulgação de informes financeiros que disseminam um cabedal de conhecimento de fácil acesso. Mas, paralelo a essa acessibilidade, coexiste a responsabilidade exigida pela Lei SOX Seção 404 em que a administração deve elaborar, implementar e manter sistemas eficazes dos controles internos, bem como, o comprometimento da instituição na transparência e confiabilidade dos relatórios financeiros perante os usuários de suas informações.

Nesse contexto, o acesso à informação deve vir atrelado a padrões de confiabilidade dos informes contábeis. Portanto, os departamentos que compilam e emitem as informações devem estar qualificados para esta tarefa conforme critérios estabelecidos pelo Conselho Federal de Contabilidade (CFC) e órgãos reguladores. A automação na execução dos trabalhos de Auditoria representa uma tendência decorrente do volume e da complexidade das transações comerciais. Por exemplo, apenas o novo modelo de avião da Boeing, Dreamliner, contém mais de 500 mil peças e centenas de fornecedores. Isso faz com que somente através de um processo extremamente informatizado, atualizado em tempo real e automatizado (Auditoria Contínua) seja capaz de avaliar controles e riscos em bases contínuas e independentes, de forma a identificar exceções e anomalias, tendências e indicadores de risco na intenção de fornecer garantias financeiras em consonância ao plano estratégico e operacional da instituição.

Segundo Tostes (2007), a partir dos anos 1990, houve significante aumento de transações no mercado financeiro de instrumentos derivativos. Os preços de produtos como opções e futuros oscilavam mais rápidos e intensamente do que mercados a vista. Por serem os produtos derivativos mais voláteis, a redução de risco por meio de diversificação não tem sido suficiente como nos mercados tradicionais. A precificação a custo histórico cedeu lugar à avaliação de posições mark-to-market com frequentes reavaliações e foco para captar, instantaneamente o efeito potencial das alterações de valor nas posições assumidas em carteiras de investimento. O autor refere-se, também, a alterações do paradigma quanto ao valor dos instrumentos derivativos, provocando um aumento no número de transações e lançamentos contábeis. O método de amostragem estatística em contas de saldo com alto valor e número de transações na faixa de milhões, tornou-se impraticável. Por mais flexível que sejam os critérios, o tamanho de amostra $(\mathrm{N})$ irá conter centenas ou milhares de operações impossíveis de serem examinadas em detalhe por indivíduos auditores.

A aceleração dos fluxos de informação e a disponibilidade de sistemas on-line em tempo real levou a profissão contábil a repensar o significado da auditoria e o seu processo de trabalho. Atualmente, acredita-se que o método de visitas periódicas de auditor externo onde ele opina ex-post - é uma relíquia da era pré-digital (ALLES; TOSTES; VASARHELYI; e RICCIO, 2006).

Dentre alguns fatores que favoreceram a evolução da auditoria contínua em ambiente computadorizado, destacam-se a crescente demanda por sistemas sofisticados que acompanhem o grande volume de processamento de dados; a complexidade e competência técnica indispensáveis na gestão dos dados, bem como, o atendimento às exigências regulamentares.

Atualmente, existe uma tendência no uso da Auditoria Contínua integrada com os sistemas de gestão corporativos ERP (Enterprise Resource Planning), que ao utilizar uma mesma base de dados e linguagens de programação padronizadas XML (Extensible Markup Language) e XBRL (Extensible Business Reporting Language) tornam possível a consolidação das informações financeiras com mais segurança e menos riscos, por meio de relatórios via Web, com a frequência calculada para disponibilização em tempo real. 
Diante do exposto, elaborou-se a seguinte questão de pesquisa: Quais as tendências de pesquisa sobre a Auditoria Contínua evidenciadas em periódicos brasileiros com classificação Qualis Capes A2, B1, B2 e B3 no período de 2006 a 2011 ?

Nesse cenário, o presente estudo tem por objetivo investigar tendências de pesquisa sobre Auditoria Contínua em artigos publicados em periódicos brasileiros com classificação A2, B1, B2 e B3, no período de 2006 a 2011. Como objetivo específico, pretende-se destacar os conceitos desenvolvidos na Auditoria Contínua.

Este estudo divide-se em seis seções. A introdução apresenta o problema de pesquisa e os objetivos do estudo. A segunda aborda a fundamentação teórica, com ênfase no contexto histórico; nos conceitos de Auditoria Contínua; na descrição das etapas necessárias para a realização da Auditoria Contínua e na apresentação sintética de pesquisas científicas de Auditoria Contínua publicadas em periódicos internacionais. A terceira seção destaca pesquisas de Auditoria Contínua publicadas em periódicos brasileiros. A quarta seção discorre sobre a metodologia adotada para o desenvolvimento das pesquisas. A quinta a descrição e análise dos dados. Encerrando, são apresentadas as considerações finais.

\section{REFERENCIAL TEÓRICO}

Esta seção apresenta o contexto histórico; definição de autoria contínua e as etapas necessárias para sua realização e as pesquisas científicas publicadas em periódicos internacionais sobre o assunto.

\subsection{ASPECTOS HISTÓRICOS E CONCEITUAIS}

O conceito de "Auditoria Integral" teve origem nos anos 1990. Conforme Nasi (1997), o trabalho com uma abordagem ampla de atuação nas auditorias operacional, administrativa, financeira e de qualidade representava um incremento da auditoria em atender às expectativas do mercado no gerenciamento das informações.

A princípio, com a intenção de proporcionar à empresa auditada uma gestão eficaz em seus negócios, a Auditoria Integral surgiu como uma opção à auditoria tradicional da época, com ênfase na apresentação de dados concisos, oportunos e úteis no processo decisório da administração da entidade (FORMIGA; ALVES; MARQUES, 2004).

A Auditoria Integral seria responsável por emitir parecer sobre diversas áreas afins da instituição, que provavelmente os profissionais de auditoria não teriam competências e conhecimentos adquiridos ou desenvolvidos. Desta forma, a garantia de eficiência, efetividade, economicidade, moderação, ética e preservação do meio ambiente de seus produtos, via relatórios de auditoria, não seriam atingidas segundo Voss (1999). De acordo com o autor, o auditor não faz parte do processo de gestão dos negócios, sua responsabilidade consiste na emissão de parecer que assegure a veracidade das informações fornecidas pela instituição, em seus relatórios financeiros anuais. A Auditoria Integral caiu em desuso a partir do ano de 2000.

Após uma série de escândalos financeiros, com destaques a Enron, WorldCom e Tyco, houve um crescente aumento na preocupação por parte dos investidores e do Congresso norteamericano, quanto à execução dos trabalhos e qualidade dos relatórios de auditoria. Procurava-se evidenciar o trabalho executado nos controles internos, assegurando sua confiabilidade, base para emissão do relatório de auditoria. Sendo assim, a lei Sarbanes \& Oxley, em 2002, provocou alterações importantes, de forma indireta, nos relatórios financeiros; e direta nos de auditoria; nos métodos de controle interno e no estabelecimento de padrões e governança corporativa (ALLES; KOGAN; VASARHELYI, 2004). 
A lei Sarbanes Oxley contribuiu para a adoção de padrões e fiscalização dos controles gerenciais, e aprimoramento dos relatórios de divulgação das demonstrações financeiras. As responsabilidades dos gestores e da empresa de auditoria foram delimitadas e a aplicação das penalidades tornou-se mais rígida e abrangente, principalmente, sobre à atuação profissional dos auditores e diretoria executiva. Alles, Kogan e Vasarhelyi (2004) sugerem o uso de novas tecnologias para atender a crescente e complexa demanda por informações como preconiza a lei Sarbanes Oxley. A utilização dos sistemas computacionais propicia uma verificação contínua dos controles internos da empresa.

A Seção 404 da Lei Sarbanes Oxley determina uma avaliação anual dos controles internos para a emissão de relatórios financeiros. Também cria a necessidade de emissão de relatórios distintos que ateste a eficácia dos controles internos e dos procedimentos executados para a emissão dos relatórios financeiros.

O processo de pesquisa em auditoria contínua originou-se em 1989, acompanhando os desdobramentos iniciais da auditoria em EDP (Processamento Eletrônico dos Dados). Conforme Alles, Kogan e Vasarhelyi (2002), a primeira aplicação foi desenvolvida no Bell Laboratories da AT\&T no mesmo ano do início dos estudos, em 1989.

Groomer e Murthy (1989) propuseram módulos de auditoria, embutidos, para capturar informações em uma base contínua. Estes módulos foram desenvolvidos para capturar e avaliar os dados, sendo considerados essenciais na arquitetura de Garantia Contínua de Dados e, progressivamente, deveriam ser complementados por sistemas automatizados exercendo algumas funções limitadas de auditoria. Nesse primeiro instante o uso contínuo de análises automatizadas objetivava obter conclusões sobre uma determinada área ou atividade considerando seu risco inerente.

De acordo com Kogan, Sudit e Vasarhelyi (1999), a Auditoria contínua é um procedimento contábil que produz resultados de auditoria, simultaneamente, em um curto período de tempo após a ocorrência de eventos relevantes. Embora esta definição reflita o significado comumente aceito de auditoria contínua, alguns contadores preferem chamá-la de auditoria instantânea, em vez de contínua. A confusão surge porque em muitos casos, a auditoria instantânea leva a produzir resultados de auditoria em frequência muito alta, gerando um fluxo de resultados contínuos. No entanto, uma auditoria contínua, utilizando a definição de instantânea, pode produzir poucos resultados se os eventos relevantes ocorrerem em longos espaços de tempo, ou seja, os resultados gerados são proporcionais aos eventos relevantes ocorridos.

As mudanças nos negócios e no ambiente de auditoria sugerem que os relatórios anuais tradicionais não atendem mais as necessidades dos usuários. Os sistemas contábeis em tempo real, os relatórios financeiros de forma eletrônica e auditoria contínua estão recebendo uma atenção considerável das empresas e da comunidade contábil (REZAEE; SHARBATOGHLIE; ELAM; MCMICKLE, 2002). Os estudos realizados com as quatro maiores empresas de auditoria (PriceWaterHouseCoopers, Deloitte, Ernst \& Young e KPMG) revelam que os processos avaliados foram considerados bons; no entanto, desenhados para abrigar os conceitos de relatórios anuais. Essas grandes empresas exibem uma necessidade crescente de que a auditoria caminhe em direção à adoção de relatórios financeiros baseados na Web, utilizando ferramentas e técnicas de Auditoria Contínua (SEARCY; WOODROOF; BRUCE, 2002).

Pode-se afirmar que a Auditoria Contínua está ainda na sua fase inicial, no Brasil, devido à pequena literatura em língua portuguesa e a carência de auditores. Para Marion (2003), o Brasil é um dos países menos auditados do mundo, existindo uma intensa defasagem de auditor para cada habitante; em média, um auditor independente para um grupo de 25.000 habitantes, enquanto que na Holanda a proporção é de um auditor para um grupo de 900 . Nos Estados Unidos, estes números são de um auditor para cada grupo de 2.300 habitantes. 
Para a adoção de Auditoria Contínua, faz-se necessário o acompanhamento tecnológico dos sistemas informatizados. Segundo afirmam Alles, Tostes, Vasarhelyi e Riccio (2006), os problemas tecnológicos, as mudanças nos cenários dos negócios, como também a demanda de auditores podem representar tanto uma dificuldade quanto um incentivo a sua adoção. Verifica-se que, atualmente, existe suporte tecnológico para acompanhar os procedimentos de Auditoria Contínua. Entretanto, esta prática é restrita a empresas nas suas operações mais relevantes. Isso se deve, principalmente, aos custos elevados e ao envolvimento da alta administração no processo e desenvolvimento profissional do auditor em tecnologias e processos negociais.

\subsection{ETAPAS NECESSÁRIAS PARA REALIZAÇÃO DA AUDITORIA CONTÍNUA}

Para se obter melhor resultado sobre os controles oferecidos pela Auditoria Contínua, faz-se necessário seguir etapas básicas conforme demonstrado na Figura 1, a seguir:

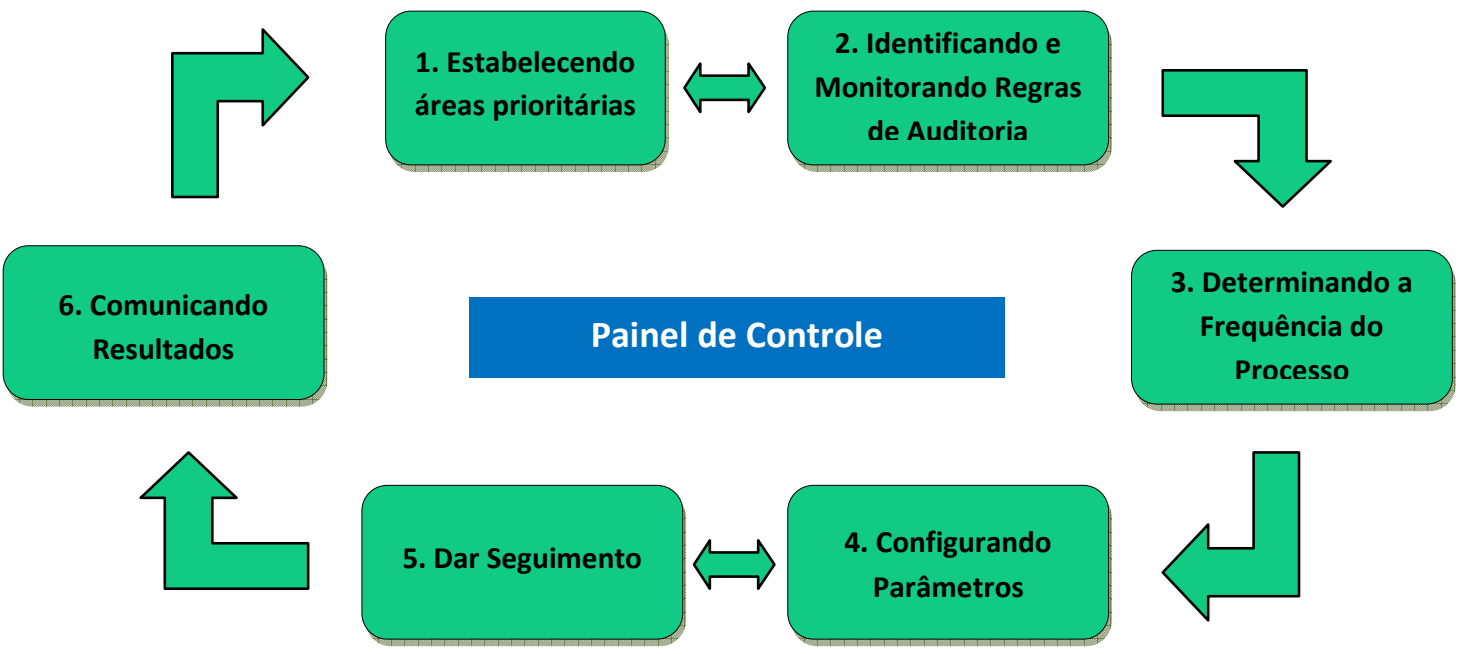

Figura 1 - Painel de Controle contendo as etapas necessárias para a realização da Auditoria Contínua. Fonte: Aquino, Silva, Sigolo e Vasarhelyi (2008)

$\mathrm{Na}$ primeira etapa, as áreas prioritárias são identificadas por meio de atividades organizacionais de auditoria que devem estar integradas como parte do plano de auditoria interna anual e o plano de gerência de risco da empresa. É, também, neste ponto que tanto os auditores internos quanto os gestores necessitam constatar se os processos críticos do negócio precisam ser auditados, classificando-os por áreas de risco e avaliando a relação custobenefício da implantação da Auditoria Contínua para cada área de risco, em particular. É nesse momento que, também, devem ser consideradas as possíveis implicações para o negócio como um todo e para uma determinada área específica, priorizando as áreas que fornecem as demonstrações de resultados mais rápidos e que agreguem valor para a empresa.

O segundo passo consiste em determinar as regras que nortearão as atividades de Auditoria Contínua e as rotinas que necessitam ser programadas e reconfiguradas. Essas regras precisam obedecer aos aspectos legais e questões do ambiente cultural da empresa, bem como, atender os objetivos do processo, em particular.

Em seguida, faz-se necessário considerar alguns aspectos importantes para determinar a frequência de um processo de auditoria contínua, a saber: o ritmo do processo a ser auditado; tempo do processo de negócio e disponibilidade de auditores treinados em análise de sistemas. 
A quarta etapa foi planejada para configurar as regras utilizadas em cada área antes do processo de auditoria contínua ser adotado. Posteriormente, a frequência de cada parâmetro deve ser alterada caso haja mudanças decorrentes da atividade a ser auditada.

Outra etapa importante consiste no follow-up que irá determinar como serão tratados os alarmes e as detecções de erros gerados pelo sistema. Nesse estágio, estão definidas as responsabilidades do auditor e o direcionamento dos eventos não tratados pelos profissionais da auditoria contínua para níveis superiores, previamente autorizados e capacitados para o acompanhamento das irregularidades apuradas, conforme o risco e prioridade de cada alarme.

A etapa final define como lidar com os resultados auditados, seja com falta de concordância detectada na auditoria ou com as considerações individuais levantadas. É importante uma análise concisa e objetiva sobre as discrepâncias de fatos se revelados nos resultados finais.

\subsection{PESQUISAS CIENTÍFICAS DE AUDITORIA CONTÍNUA}

A articulação do presente artigo decorre da análise de trabalhos com abordagens semelhantes publicados em periódicos internacionais. Os trabalhos selecionados, que contribuíram para o delineamento deste artigo, são descritos a seguir de forma sintética.

\begin{tabular}{|c|c|c|}
\hline Título & Autores & Data \\
\hline $\begin{array}{l}\text { Continuous online auditing as a response to the Sarbanes- } \\
\text { Oxley Act }\end{array}$ & EL-MASRY, E. E; RECK, J. L. & 2008 \\
\hline $\begin{array}{l}\text { The Development of a Computer Auditing System } \\
\text { Sufficient for Sarbanes-Oxley Section } 404 \text { - A Study on } \\
\text { the Purchasing and Expenditure Cycle of the ERP System }\end{array}$ & CHANG, S.; WU, C. C.; CHANG, I. & 2008 \\
\hline $\begin{array}{l}\text { An Investigation into the Application of Continuous } \\
\text { Online Auditing in the U.K.. }\end{array}$ & OMOTESO K.; PATEL A.; SCOTT P. & 2008 \\
\hline $\begin{array}{l}\text { Continuous auditing for } \text { web-released financial } \\
\text { information }\end{array}$ & CHOU, C. C.; CHANG, C. J. & 2010 \\
\hline Auditing in enterprise system environment: a synthesis & KANELLOU, A.; SPATHIS, C. & 2011 \\
\hline $\begin{array}{l}\text { Determining expected behavior of fraudsters for a } \\
\text { continuous audit system. }\end{array}$ & THOMAS, M. A.; MARATHE, R. R. & 2012 \\
\hline
\end{tabular}

El-Masry e Reck (2008) analisaram a percepção dos investidores sobre a utilidade da auditoria contínua, antes e depois da Lei Sarbanes Oxley (SOX) e avaliaram a relevância do valor atual da Auditoria Contínua. Foram conduzidos experimentos em laboratório entre os participantes, confrontando o uso da Auditoria Contínua On-Line com os riscos tecnológicos, bem como, a análise da Auditoria Contínua antes e depois da SOX. Resultados indicam uma demanda pela Auditoria Contínua, fato refletido na redução da estimativa de risco por parte dos investidores, aumentando assim a confiança nessas estimativas. Resultados comparativos da amostra de 2002 e 2005 indicam que o valor de relevância da Auditoria Contínua aumentou após a introdução de SOX em Julho de 2002. Segundo os autores, ainda são necessários outros estudos para medir a capacidade da auditoria contínua na redução da estimativa de risco considerando outros aspectos, além dos financeiros, para detectar os riscos em negócios tradicionais.

Os autores She-I Chang, Wu e I-Cheng Chang (2008) propõem o desenvolvimento de um sistema gerenciável de auditoria automatizado capaz de dar subsídio a pesquisadores e empresários sobre as tendências desta área. Este artigo foi desenvolvido levando-se em conta três quesitos: explorar os itens de controle de extrema relevância no ciclo de compras e despesas confrontando com a seção 404 da Lei SOX; desenvolver um sistema computacional de auditoria baseado nos itens de controle conhecidos e em conformidade com a Seção 404 da 
lei SOX; e, validar a aplicabilidade deste sistema de acordo com o modelo ISO/IEC 9126 para atender as necessidades organizacionais. Conclui-se que para atender os requisitos da SOX 404, que preconiza a construção, implementação e manutenção da Auditoria Interna sobre os Relatórios Financeiros, se faz necessário combinar os conhecimentos de Auditoria e Tecnologia da Informação para obter melhores resultados. O sistema se mostrou capaz de gerenciar e identificar declarações incorretas e atividades fraudulentas.

Chou e Chang (2010) mostraram que apesar da crescente oferta de informações financeiras através de relatórios na web e do ganho de comunicação que esta oferta traz para a relação entre as empresas e os usuários destes relatórios, essas informações disponibilizadas necessitam ser auditadas. Ao objetivar a proposição de novos conceitos e técnicas, esse estudo comparou a eficiência econômica de três abordagens de auditoria combinando vários tipos de divulgação, percepções de materialidade e ambientes de informação. Uma estrutura técnica baseada em uma estrutura conceitual especifica procedimentos genéricos para realizar os testes de controle online sobre os relatórios disponibilizados via web. Conclui-se que mesmo ainda necessitando de mais suporte empírico para este modelo, existem sustentações antecipadas afirmando que a Auditoria Contínua é o método mais apropriado para auditar os relatórios disponibilizados na web por possuir um modelo comportamental que simula várias informações de descoberta e auditoria do ambiente.

Kanellou e Spathis (2011) realizaram uma revisão de literatura baseada em pesquisas anteriores relacionando a Auditoria e Sistemas Corporativos, identificando temas ainda não abordados, propondo o direcionamento para pesquisas futuras e ajudando os pesquisadores sobre como sintetizar melhor essas duas áreas. Nesse estudo foram incluídos 31 trabalhos acadêmicos, que foram divididos em cinco categorias: o futuro da Auditoria no ambiente dos Sistemas Corporativos; modernas técnicas e ferramentas de Auditoria; mudança do papel do auditor; diferenças nas percepções dos auditores financeiros e de TI e sistemas ERP, em conformidade com os regulamentos. Como resultado, descobriu-se que a consolidação de um sistema corporativo resulta na reengenharia dos processos de Auditoria e aumenta a necessidade de monitoramento das transações. Foi considerada como crítica a presença do Auditor de TI no processo de desenvolvimento do sistema, bem como, falha a figura do Auditor Financeiro em transferir seu conhecimento na condução de testes efetivos de auditoria. Modernas técnicas e ferramentas de auditoria também se fazem necessárias para a implantação de um sistema corporativo integrado com a auditoria.

Thomas e Marathe (2012) determinaram o comportamento dos fraudadores através de um modelo probabilístico em que o fraudador é racional e as consequências de suas ações devem ser consideradas antes de sua ação ser completada. Foi desenvolvido um modelo com vetores que avalia as ações e intenções do fraudador e como a auditoria contínua pode ser utilizada para assegurar a não conformidades destes atos. $\mathrm{O}$ modelo probabilístico nos permite calcular o comportamento esperado de um fraudador em um sistema de transação, desta maneira, permite a auditoria contínua decidir o tempo e frequência de um sistema na detecção de fraudes.

O estudo, a seguir, refere-se a pesquisa exploratória utilizando método quantitativo. Omoteso, Patel e Scott (2008) indicaram que auditores têm adotado, cada vez mais, as tecnologias online na execução de suas tarefas. Como a maioria dos estudos sobre tecnologia online na área de Auditoria não tem sido de natureza empírica, esse artigo vem trazer esse foco de investigação, sinalizando as vantagens e desvantagens da Auditoria Contínua Online, avaliando sua relevância para o futuro da auditoria tanto interna quanto externa. Como conclusão, o artigo traz algumas reflexões sobre impactos na expansão da Auditoria Contínua Online.

Verifica-se que as publicações estrangeiras (Quadro 1) sobre a Auditoria Contínua adotaram enfoques diferenciados. Nos últimos anos, com a crescente utilização da Tecnologia da 
Informação e dos sistemas integrados de ERP, houve uma demanda natural para a migração dos conceitos de auditoria tradicional para a Auditoria Contínua Online.

Os artigos sobre Auditoria Contínua publicados no Brasil foram pesquisados na Revista Gestão de Tecnologia e Sistemas de Informação pela Universidade de São Paulo (USP) classificada pela Qualis Capes, como B1. A seguir, os artigos são descritos de forma breve.

\begin{tabular}{|c|c|c|}
\hline Título & Autores & Data \\
\hline $\begin{array}{l}\text { Continuous Auditing: The USA Experience and } \\
\text { Considerations for its Implementation in Brazil. }\end{array}$ & $\begin{array}{l}\text { ALLES, M. G.; TOSTES, F. } \quad \text { P.; } \\
\text { VASARHELYI, M. A.; RICCIO, E. L. }\end{array}$ & 2006 \\
\hline $\begin{array}{l}\text { Integrating Analytical Procedures into the } \\
\text { Continuous Audit Environment. }\end{array}$ & KOSKIVAARA, E. & 2006 \\
\hline $\begin{array}{l}\text { Resultados do } 7^{\circ} \text { CONTECSI }- \text { Congresso } \\
\text { Internacional de Gestão da Tecnologia e Sistemas de } \\
\text { Informação. }\end{array}$ & $\begin{array}{l}\text { RICCIO, E. L.; SAKATA, M. C. G.; } \\
\text { VALENTE, N. T. Z. }\end{array}$ & 2010 \\
\hline
\end{tabular}

Alles, Tostes, Vasarhelyi, Riccio (2006) discutiram sobre a experiência americana no uso da Auditoria Contínua e as considerações para sua implementação no Brasil. Por meio da revisão de literatura, o estudo demonstrou a evolução da Auditoria Contínua nos EUA, os aspectos que impulsionaram sua utilização com destaques as determinações da Lei SOX.

Para adotar a Auditoria Contínua no Brasil, deve haver uma reformulação nos aspectos jurídicos e um aumento na quantidade de auditores. $\mathrm{O}$ estudo indica o interesse por parte dos executivos na participação de congressos especializados, na busca de material de estudo e na contratação de consultores especializados, principalmente no setor bancário e de telecomunicações para avaliar o impacto e o custo de implantação da Auditoria Contínua em suas empresas. O artigo aborda a conceituação de Auditoria Contínua, de Monitoramento e Controle Contínuo, apresentando um comparativo entre a Auditoria Tradicional e a Auditoria Contínua, além de apontar os benefícios na gestão dos negócios com ênfase na confiabilidade e temporariedade dos dados, destaca as principais dificuldades encontradas no cenário brasileiro (falta de literatura nacional, número reduzido de auditores e custo elevado em tecnologia). Pode-se concluir pelos estudos apresentados neste artigo que a Auditoria Contínua tende a uma evolução natural, como foi nos processos de transição dos sistemas ERP, sendo possível realizar a automação e integração dos sistemas e processos previstos pela Auditoria Contínua (ALLES; TOSTES; VASARHELYI; RICCIO, 2006).

KOSKIVAARA (2007) apresentou estudo sobre como integrar procedimentos analíticos no ambiente de Auditoria Contínua. Utilizando diferentes técnicas de procedimentos analíticos, o autor demonstrou a importância do seu uso na Auditoria Contínua e concluiu que o uso de redes neurais e de regressão são técnicas de bom resultado. Destaca que o sucesso na aplicação da Auditoria Contínua está diretamente relacionado com treinamentos, softwares, sistema de segurança em níveis adequados e das habilidades do auditor no entendimento dos sistemas de informação e das técnicas estatísticas utilizadas.

Riccio, Sakata, Valente (2010) trazem os resultados do $7^{\circ}$ CONTECSI, Congresso Internacional de Gestão da Tecnologia e Sistemas de Informação. O estudo apresentou dados estatísticos, justificativos e objetivos do evento, programa, sessões plenárias, informações sobre o $20^{\circ}$ Simpósio Anual de Auditoria Contínua, sessões paralelas, menção honrosa, comitês e alguns destaques. Esta conferência é um dos poucos eventos que trata da Auditoria Contínua no Brasil, e na edição de 2010, trouxe um total de 233 trabalhos em 47 sessões, discutindo os efeitos da Tecnologia e dos Sistemas de Informação na sociedade e nas organizações. O que houve de mais relevante para o estudo da Auditoria Contínua neste evento foi o $20^{\circ}$ Simpósio Mundial sobre Auditoria Contínua, trazendo para a sessão assuntos 
de interesse profissionais e acadêmicos como: um estudo de caso da Auditoria Contínua no Itaú-Unibanco e no Banco Santander; Tendências identificadas em projetos de Auditoria Contínua; O papel da Auditoria de Sistemas na Nova Economia; Tendências e certificações profissionais sobre o tema.

Verifica-se que as publicações sobre a Auditoria Contínua no cenário nacional (Quadro 2) descrevem conceitos, técnicas, vantagens, dificuldades e aspectos gerais da implementação da Auditoria Contínua. No período pesquisado não foram encontrados estudos empíricos em periódicos brasileiros com empresas nacionais que adotam a Auditoria Contínua e a respectiva correlação desta técnica com outros indicadores financeiros e não financeiros.

\section{METODOLOGIA DE PESQUISA}

Considerando o objetivo proposto, o procedimento metodológico adotado foi o da pesquisa exploratória. Segundo Collis e Hussey (2005), a investigação exploratória é realizada sobre um problema de pesquisa com pouco ou nenhum estudo anterior. O propósito desta pesquisa é procurar por padrões ou ideias. Não há na pesquisa exploratória testes de hipóteses, ou seja, as proposições não são certificadas pela associação ou causalidade. $O$ foco da pesquisa exploratória consiste em fornecer dados preliminares para o entendimento e percepção do tema, sendo posteriormente desenvolvida uma investigação mais profunda e metodológica. Geralmente, este tipo de pesquisa concentra-se em obter uma quantidade elevada de informações e, frequentemente, não responde com precisão ao problema proposto. Entretanto, pode indicar o desenvolvimento de estudos pertinentes.

A Coordenação de Aperfeiçoamento de Pessoal de Nível Superior (CAPES) com o objetivo de expandir e consolidar os cursos de pós-graduação stricto sensu desempenha atividades relacionadas com o acesso e divulgação de estudos científicos, como também investe em recursos nos programas junto às instituições de ensino, alunos e professores. Um dos procedimentos adotados para garantir a qualidade da produção intelectual é a classificação anual de periódicos por áreas de avaliação, disponibilizando, no seu site, o Qualis Capes de acordo com os indicativos dos estratos em A1, (o mais elevado com 100 pontos) A2 (80 pontos), B1 (60 pontos), B2, (50 pontos), B3 (30 pontos), B4 (20 pontos), B5 (10 pontos) e C (não possui pontuação).

Atualmente, o Qualis Capes da área de Administração, Contabilidade e Turismo contempla o total de 1.503 periódicos distribuídos pelas variadas pontuações anteriormente descritas. Desta maneira, a partir de uma abordagem exploratória, este estudo buscou a identificação e compilação dos trabalhos publicados sobre a Auditoria Contínua em periódicos brasileiros A2, B1, B2 e B3, no período de 2006 a 2011. O período de seis anos foi escolhido porque o ano de 2006 marca o início de publicações em periódicos nacionais e a ocorrência da primeira conferência na Universidade de São Paulo (USP) sobre o tema no Brasil e, em paralelo, busca-se por tendências deste assunto no contexto atual.

O levantamento de dados, para esta pesquisa, ocorreu obedecendo ao seguinte padrão:

a) Busca do tema em periódicos internacionais e nacionais;

b) Seleção de periódicos nacionais conforme a avaliação Qualis Capes A2, B1, B2 e B3 concentrados nas áreas de atuação: Administração, Ciência da Informação, Ciências Sociais, Contabilidade, Economia, Engenharia de Produção, Finanças, Gestão de Tecnologia e Sistema de Informação e Gestão e Estratégica/Desenvolvimento.

c) Seleção de trabalhos centrando a busca pelas palavras-chaves: Auditoria, Auditoria Contínua, Auditoria Digital e Relatório Financeiro On-line;

d) Leitura dos artigos e sua classificação, conforme abordagem adotada sobre o tema da Auditoria Contínua; 
e) Leitura técnica sobre a metodologia adotada e coleta de dados;

f) Elaboração de catálogo com os dados levantados (ano, tema, método de coleta, título, autores, periódicos e bibliografia).

Esta coleta de dados ocorreu entre os meses de abril a junho de 2012 e logo após depurações, obteve-se a amostra para este estudo de 121 periódicos nacionais e seis periódicos internacionais. Sendo assim, foram identificados os artigos nacionais sobre a Auditoria Contínua bem como, apuradas suas tendências no contexto brasileiro em virtude das suas práticas no ambiente internacional.

\section{APRESENTAÇÃO E ANÁLISE DOS RESULTADOS}

O universo pesquisado refere-se aos artigos publicados sobre a Auditoria Contínua, em periódicos brasileiros A2, B1, B2 e B3, no período de 2006 a 2011. O estudo também abordou algumas pesquisas publicadas em periódicos internacionais, pois assim, obtêm-se um parâmetro para verificar como o tema tem sido desenvolvido e identificar, avaliar e comparar as tendências de pesquisas brasileiras em Auditoria Contínua.

Foram utilizadas as referências contidas no quadro 3, a seguir, no apoio e desenvolvimento deste estudo.

\begin{tabular}{|c|c|c|c|c|}
\hline Autor & Título & $\begin{array}{l}\text { Questão de } \\
\text { Pesquisa }\end{array}$ & Resultados & $\begin{array}{l}\text { Sugestões para } \\
\text { Investigação }\end{array}$ \\
\hline $\begin{array}{l}\text { Miclhael } \\
\text { Alles; } \\
\text { Alexander } \\
\text { Kogan e } \\
\text { Miklos } \\
\text { Vasarhelyi }\end{array}$ & $\begin{array}{l}\text { A Lei das } \\
\text { Consequências } \\
\text { não } \\
\text { Intencionais? }\end{array}$ & $\begin{array}{l}\text { Avaliar os } \\
\text { Custos, } \\
\text { Benefícios e } \\
\text { Resultados da } \\
\text { Lei Sarbanes- } \\
\text { Oxley. }\end{array}$ & $\begin{array}{l}\text { A lei SOX prevê } \\
\text { controles internos } \\
\text { confiáveis e em tempo } \\
\text { oportuno. }\end{array}$ & $\begin{array}{l}\text { Uso de novas } \\
\text { tecnologias para } \\
\text { acompanhar a } \\
\text { complexidade das } \\
\text { operações da empresa } \\
\text { com menores custos e } \\
\text { maior capacidade. }\end{array}$ \\
\hline $\begin{array}{l}\text { SERRA, } \\
\text { Sara }\end{array}$ & $\begin{array}{l}\text { A Divulgação } \\
\text { de Informação } \\
\text { Financeira On- } \\
\text { Line: Um } \\
\text { desafio para a } \\
\text { Auditoria. }\end{array}$ & $\begin{array}{l}\text { Conhecer a } \\
\text { situação da } \\
\text { Auditoria } \\
\text { perante relato } \\
\text { financeiro on- } \\
\text { line. }\end{array}$ & $\begin{array}{l}\text { Necessidade de } \\
\text { mudança tanto em } \\
\text { relação à divulgação } \\
\text { quanto no perfil dos } \\
\text { auditores para garantir } \\
\text { segurança e veracidade } \\
\text { nas informações. }\end{array}$ & $\begin{array}{l}\text { A auditoria e os seus } \\
\text { profissionais devem } \\
\text { ultrapassem os } \\
\text { desafios da nova } \\
\text { realidade. }\end{array}$ \\
\hline $\begin{array}{l}\text { NIGRINI, } \\
\text { Mark J. }\end{array}$ & $\begin{array}{l}\text { Continuous } \\
\text { Auditing }\end{array}$ & $\begin{array}{l}\text { Analisar bases } \\
\text { digitais que } \\
\text { possam ser } \\
\text { utilizadas pelos } \\
\text { auditores. }\end{array}$ & $\begin{array}{l}\text { Pareceres e riscos de } \\
\text { auditoria são movidos } \\
\text { por distorções } \\
\text { materiais relevantes. }\end{array}$ & $\begin{array}{l}\text { Pesquisas com testes } \\
\text { de hipóteses usados } \\
\text { nas novas tecnologias } \\
\text { que assegurem a } \\
\text { qualidade do trabalho } \\
\text { de auditoria. }\end{array}$ \\
\hline $\begin{array}{l}\text { TANK, } \\
\text { Koen } \\
\text { Klein }\end{array}$ & $\begin{array}{l}\text { Continuous } \\
\text { Auditing \& } \\
\text { Continuous } \\
\text { Monitoring in } \\
\text { a Broader } \\
\text { Perspective }\end{array}$ & $\begin{array}{l}\text { Como fazer CA } \\
\& \text { CM } \\
\text { interessante para } \\
\text { a gestão das } \\
\text { organizações }\end{array}$ & $\begin{array}{l}\text { Desde que sejam } \\
\text { realizadas melhorias, a } \\
\text { organização pode } \\
\text { obter potencial para o } \\
\text { CA \& CM e, em } \\
\text { consequência, agregar } \\
\text { valor a estratégia para } \\
\text { gerir riscos e aumentar } \\
\text { o desempenho. }\end{array}$ & $\begin{array}{l}\text { Novas pesquisas com } \\
\text { diferentes ferramentas, } \\
\text { metodologias, } \\
\text { frameworks, empresas } \\
\text { e processo que possam } \\
\text { ser mais úteis na } \\
\text { gestão de } \\
\text { desempenhos. }\end{array}$ \\
\hline
\end{tabular}

Quadro 3 - Artigos com a temática Auditoria Contínua.

Fonte: Dados dos autores, 2012. 
A bibliografia indicada no quadro acima foi útil na construção desta pesquisa, pois permitiu o conhecimento da aplicação da Auditoria Contínua e seu impacto nas estratégias das organizações.

\subsection{PUBLICAÇÃO POR PERIÓDICOS}

A base de pesquisa utilizou os periódicos brasileiros do documento de área de 2009 dos cursos de Administração, Ciências Contábeis e Turismo, que são agrupados, avaliados e disponíveis de acordo com os critérios adotados pela Capes. A escolha deste documento devese ao fato de que a Auditoria é um segmento de atuação profissional e/ou acadêmica da Ciência Contábil. Com isso, foram selecionadas as áreas afins com Auditoria e em seguida, Auditoria Contínua. Posteriormente, foram adotadas a avaliação Qualis em A2, B1, B2 e B3. Por meio da identificação dos periódicos em conformidade com suas áreas de atuação, podese obter um universo mais abrangente da pesquisa quanto à busca de artigos em Auditoria Contínua. Após a correlação das ciências afins com Auditoria Contínua, o levantamento dos dados adotou os periódicos brasileiros descritos nos quadros 4, 5, 6 e 7 segundo a avaliação Qualis Capes respectivamente em A2, B1, B2 e B3.

\begin{tabular}{|l|l|c|}
\hline \multicolumn{1}{|c|}{ ISSN } & \multicolumn{1}{|c|}{ Titulo } & Total \\
\hline $1807-7692$ & BAR. Brazilian Administration Review & 108 \\
\hline $1807-734 X$ & BBR. Brazilian Business Review & 108 \\
\hline $0011-5258$ & Dados (Rio de Janeiro. Impresso) & 173 \\
\hline $0104-530 X$ & Gestão \& Produção (UFSCAR. Impresso) & 307 \\
\hline $1984-9230$ & Organizações \& Sociedade (Online) & 232 \\
\hline $1982-7849$ & RAC. Revista de Administração Contemporânea & 281 \\
\hline $1676-5648$ & RAE Eletrônica (Online) & 67 \\
\hline $0034-7140$ & Revista Brasileira de Economia (Impresso) & 142 \\
\hline $1808-057 X$ & Revista Contabilidade \& Finanças (Online) & 129 \\
\hline $0034-7612$ & Revista de Administração Pública (Impresso) & 290 \\
\hline & TOTAL DE PUBLICAÇÕES A2 & $\mathbf{1 8 3 7}$ \\
\hline
\end{tabular}

Quadro 4 - Periódicos brasileiros A2.

Fonte: CAPES, 2012.

O quadro 4 apresenta o total de 10 periódicos. Observa-se um reduzido número de periódicos caracterizados pela Qualis Capes como A2 quando comparados com as qualificações B1, B2 e B3. Possivelmente, justificado pelas exigências e padrões de indexação internacionais necessários para criar e manter periódicos com os requisitos determinados pela Capes.

\begin{tabular}{|l|l|c|}
\hline \multicolumn{1}{|c|}{ ISSN } & \multicolumn{1}{|c|}{ Titulo } & Total \\
\hline $1983-8239$ & Caderno CRH (Online) & 91 \\
\hline $1679-3951$ & Cadernos EBAPE.BR (FGV) & 278 \\
\hline $0103-734 X$ & Contabilidade Vista \& Revista & 139 \\
\hline $1413-8050$ & Economia Aplicada (Impresso) & 166 \\
\hline $0104-0618$ & Economia e Sociedade (UNICAMP. Impresso) & 111 \\
\hline $0873-7444$ & Economia Global e Gestão & 80 \\
\hline $1518-2924$ & Encontros Bibli & 188 \\
\hline $0104-4036$ & Ensaio (Fundação Cesgranrio. Impresso) & 161 \\
\hline $0101-4161$ & Estudos Econômicos (São Paulo. Impresso) & 191 \\
\hline
\end{tabular}




\begin{tabular}{|c|c|c|}
\hline $1809-4783$ & Informação \& Sociedade (UFPB. Online) & 87 \\
\hline $1518-7012$ & Interações (UCDB) & 87 \\
\hline 0024-7413 & Luso-Brazilian Review & 83 \\
\hline 0101-3300 & Novos Estudos CEBRAP (Impresso) & 161 \\
\hline $0103-863 X$ & Paidéia (USP. Ribeirao Preto. Impresso) & 217 \\
\hline $1413-9936$ & Perspectivas em Ciência da Informação & 218 \\
\hline $0101-7438$ & Pesquisa Operacional (Impresso) & 185 \\
\hline 0103-7307 & Pró-Posições (UNICAMP. Impresso) & 59 \\
\hline $0103-6513$ & Produção (São Paulo. Impresso) & 281 \\
\hline $1809-2039$ & RAI : Revista de Administração e Inovação & 169 \\
\hline $1678-6971$ & RAM. Revista de Administração Mackenzie & 237 \\
\hline $1983-7488$ & RAUSP-e (São Paulo) & 186 \\
\hline $0102-3098$ & Revista Brasileira de Estudos de População & 112 \\
\hline $1679-0731$ & Revista Brasileira de Finanças (Impresso) & 99 \\
\hline $1806-4892$ & Revista Brasileira de Gestão de Negócios (São Paulo) & 135 \\
\hline $1809-239 X$ & Revista Brasileira de Gestão e Desenv. Regional & 142 \\
\hline $1679-3390$ & Revista Brasileira de Orientação Profissional & 91 \\
\hline $0080-2107$ & Revista de Administração (FEA-USP) & 176 \\
\hline $1516-3865$ & Revista de Ciências da Administração (CAD/UFSC) & 157 \\
\hline $1982-6486$ & Revista de Contabilidade e Organizações & 104 \\
\hline $1415-9848$ & Revista de Economia Contemporânea (Impresso) & 116 \\
\hline $1807-1775$ & Revista de Gestão da Tecnologia e Sistemas de Informação & 142 \\
\hline $1645-4464$ & Revista Portuguesa e Brasileira de Gestão & 36 \\
\hline $1809-3337$ & Revista Universo Contábil & 183 \\
\hline TOTAL & TOTAL DE PUBLICAÇÕES B1 & 4868 \\
\hline
\end{tabular}

Quadro 5 - Periódicos brasileiros B1.

Fonte: CAPES, 2012.

O quadro 5 informa o total de 34 periódicos classificados como B1. É verificado um número de periódicos superior à pontuação A2. Como uma das possíveis explicações para que os periódicos $\mathrm{B} 1$ estejam em maior quantidade em relação à $\mathrm{A} 2$ deve-se ao nível elevado de qualidade (nacional) que apresenta, em especial, os periódicos das renomadas instituições de ensino dos cursos stricto sensu e/ou sociedade científica reconhecida pelo comitê da área. Entretanto, não possuem o padrão internacional e os requisitos de pontuação necessários para preencher a lacuna de publicações com A2.

\begin{tabular}{|l|l|c|}
\hline \multicolumn{1}{|c|}{ ISSN } & \multicolumn{1}{|c|}{ Titulo } & Total \\
\hline $1980-6302$ & Análise (PUCRS. Online) & 98 \\
\hline $1807-054 X$ & Base (UNISINOS) & 137 \\
\hline $1518-8353$ & Ciência da Informação (Online) & 135 \\
\hline $1984-3925$ & Contabilidade, Gestão e Governança & 101 \\
\hline $1678-2089$ & Contextus (Fortaleza) & 68 \\
\hline $1984-882 X$ & Enfoque & 99 \\
\hline $1980-2668$ & Ensaios FEE (Online) & 154 \\
\hline $1516-9103$ & Gestão \& Planejamento (Salvador) & 80 \\
\hline $1981-1543$ & Gestão \& Tecnologia de Projetos & 70 \\
\hline $1518-6911$ & Ideação (Unioeste. Impresso) & 82 \\
\hline
\end{tabular}




\begin{tabular}{|l|l|c|}
\hline $1980-0193$ & Perspectivas Contemporâneas & 79 \\
\hline $1679-5830$ & Pesquisa \& Desenv. Engenharia de Produção & 63 \\
\hline $1984-6983$ & Pretexto (Belo Horizonte. Online) & 107 \\
\hline $1983-8484$ & REBRAE. Revista Brasileira de Estratégia (Impresso) & 85 \\
\hline $2178-0080$ & Revista Administração em Diálogo - RAD & 84 \\
\hline $1677-2504$ & Revista Brasileira de Inovação & 63 \\
\hline $1984-6606$ & Revista Economia \& Gestão & 94 \\
\hline $2176-0756$ & Revista Iberoamericana de Estratégia & 127 \\
\hline $1676-1901$ & Revista Produção Online & 234 \\
\hline $1983-6635$ & RGO. Revista Gestão Organizacional (Online) & 54 \\
\hline & TOTAL DE PUBLICAÇÕES B2 & $\mathbf{2 0 1 4}$ \\
\hline
\end{tabular}

Quadro 6 - Periódicos brasileiros B2.

Fonte: CAPES, 2012.

O quadro 6 apresenta o total de 20 periódicos do tipo B2. Verifica-se uma redução na quantidade de periódicos quando comparados ao nível B1. Nesse caso, é possível inferir que a quantidade de periódicos não está diretamente atribuída na avaliação Qualis Capes e sim à qualidade, ou seja, espera-se que quanto menor os padrões de exigências para avaliação, maiores o número de periódicos disponíveis, no entanto, como não houve o incremento na quantidade, percebe-se que a Capes define requisitos necessários para avaliação comprometidos com cada nível de excelência destinada às publicações.

\begin{tabular}{|c|l|c|}
\hline ISSN & \multicolumn{1}{|c|}{ Titulo } & Total \\
\hline $0103-4979$ & Caderno CRH (UFBA. Impresso) & 92 \\
\hline $1806-2261$ & Cadernos Gestão Pública e Cidadania & 30 \\
\hline $1519-7050$ & Ciências Sociais Unisinos & 91 \\
\hline $1676-6016$ & Contexto (UFRGS) & 73 \\
\hline $1517-3801$ & Datagramazero (Rio de Janeiro) & 193 \\
\hline $1678-4855$ & Desenvolvimento em Questão & 71 \\
\hline $2175-3687$ & Diálogo e Interação & 8 \\
\hline $1983-1838$ & E-tech:Tecnologias para Competitividade Industrial & 19 \\
\hline $1808-7310$ & Estudos Tecnológicos (Online) & 97 \\
\hline $1984-6975$ & Faces: Revista de Administração (Belo Horizonte) & 154 \\
\hline $2178-9010$ & GeSec: Revista de Gestão e Secretariado & 32 \\
\hline $2176-5308$ & Gestão \& Regionalidade (Online) & 130 \\
\hline $1809-0214$ & Gestão Contemporânea (FAPA) & 36 \\
\hline $2178-8030$ & Gestão e Planejamento & 78 \\
\hline $1980-5756$ & Gestão e Sociedade (UFMG) & 71 \\
\hline $2177-1243$ & Gestão Pública: Práticas e Desafios & 46 \\
\hline $1679-1827$ & Gestão.Org & 147 \\
\hline $1808-3536$ & Liinc em Revista & 125 \\
\hline $1415-5109$ & Pensamento \& Realidade & 74 \\
\hline $1519-0412$ & Pensar Contábil & 124 \\
\hline $1808-575 X$ & Perspectiva Econômica (São Leopoldo. Online) & 50 \\
\hline $0100-0551$ & Pesquisa e Planejamento Econômico & 101 \\
\hline $0103-4138$ & Planejamento e Politicas Publicas & 76 \\
\hline $1983-8026$ & Produto \& Produção (Online) & 86 \\
\hline $2178-7638$ & RACEF - Revista de Adm., Contab. e Economia da FUNDACE & 15 \\
\hline $1984-6266$ & RC\&C. Revista de Contabilidade e Controladoria & 51 \\
\hline $1413-2311$ & REAd. Revista Eletrônica de Administração & 203 \\
\hline
\end{tabular}




\begin{tabular}{|c|c|c|}
\hline $1677-7387$ & RECADM: Revista Eletrônica de Ciência Adm. & 108 \\
\hline $2177-8736$ & REGE Revista de Gestão & 198 \\
\hline 2179-734X & Registro Contábil - RECONT & 18 \\
\hline $1677-3071$ & RESI: Revista Eletrônica de Sistemas de Informação & 101 \\
\hline $2177-3726$ & Revista "Vianna Sapiens" & 34 \\
\hline $1983-7089$ & Revista ADMpg (Online) & 88 \\
\hline 1983-716X & Revista Alcance (Online) & 145 \\
\hline $2176-9036$ & Revista Ambiente Contábil & 30 \\
\hline $1518-5532$ & Revista ANGRAD & 159 \\
\hline 2179-684X & Revista Brasileira de Administração Científica & 11 \\
\hline $1516-2664$ & Revista CESUMAR & 120 \\
\hline $1807-1821$ & Revista Contemporânea de Contabilidade (UFSC) & 96 \\
\hline $1982-2537$ & Revista da Micro e Pequena Empresa (FACCAMP) & 98 \\
\hline $1983-4659$ & Revista de Administração da UFSM & 113 \\
\hline $1679-5350$ & Revista de administração da Unimep & 146 \\
\hline $1981-8610$ & Revista de Educação e Pesquisa em Contabilidade & 82 \\
\hline $2176-8854$ & Revista de Finanças Aplicadas & 23 \\
\hline $2236-0972$ & Revista de Gestão e Projetos & 32 \\
\hline $1982-3967$ & Revista de Informação Contábil (UFPE) & 109 \\
\hline $1980-4431$ & Revista de Negócios (Online) & 138 \\
\hline $1415-2061$ & Revista do CCEI & 114 \\
\hline $1984-3372$ & Revista Eletrônica de Estratégia \& Negócios & 71 \\
\hline $1677-9479$ & Revista Gestão \& Tecnologia & 90 \\
\hline $1808-0448$ & Revista Gestão Industrial & 300 \\
\hline $1982-2596$ & Revista Pensamento Contemporâneo em Administração (UFF) & 84 \\
\hline $2179-5037$ & Revista UNIABEU & 25 \\
\hline $1980-5160$ & S \& G. Sistemas \& Gestão & 118 \\
\hline 0103-0620 & Sociais e Humanas & 149 \\
\hline $1982-7342$ & Sociedade, Contabilidade e Gestão (UFRJ) & 98 \\
\hline \multirow[t]{2}{*}{$2178-3438$} & Unoesc \& Ciência - ACHS & 45 \\
\hline & TOTAL DE PUBLICAÇÕES B3 & 5216 \\
\hline
\end{tabular}

Quadro 7 - Periódicos brasileiros B3.

Fonte: CAPES, 2012.

O quadro 7 apresenta o total de 57 periódicos de qualificação B3, este número é coerente com padrões menos exigentes que as demais acima citadas, portanto, possibilita um acesso maior das instituições e órgãos científicos. O critério na adoção dos periódicos, acima, baseou-se tanto nas áreas de atuação de estudo e a avaliação QualiCapes como também na disponibilidade das informações por pesquisas on-line. Desta forma, foram excluídas as revistas com erro na página de internet e com acesso exclusivo por meio de cadastro ou assinaturas.

Apresenta-se na tabela 1, o total de periódicos e seu respectivo percentual no universo pesquisado.

Tabela 1 - Total de Periódicos Brasileiros Pesquisados Pela Qualificação CAPES

\begin{tabular}{c|c|c|}
\hline Tipo & Total & $\%$ \\
\hline A2 & 10 & $8 \%$ \\
\hline
\end{tabular}




\begin{tabular}{c|c|c}
\hline B1 & 34 & $28 \%$ \\
\hline B2 & 20 & $17 \%$ \\
\hline B3 & 57 & $47 \%$ \\
\hline Total & $\mathbf{1 2 1}$ & $\mathbf{1 0 0 \%}$ \\
\hline
\end{tabular}

Fonte: Dados dos autores, 2012.

A tabela 1 demonstra a proporção de cada classificação Qualis Capes em A2, B1, B2 e B3 em relação ao total destes periódicos no período de 2006 até 2012. Pode-se constatar que o tipo A2 apresenta uma menor participação e em contrapartida, o tipo B3 um índice elevado. Estes percentuais podem refletir os critérios adotados pela Capes na avaliação dos seguintes itens: (a) tempo de vida do periódico; (b) endogenia e pluralidade institucional dos autores; (c) vinculação a programa stricto sensu ou sociedade científica reconhecida pelo Comitê de área e (d) qualidade geral do periódico e de seus artigos publicados.

Cabe ressaltar a importância da indexação dos periódicos em bases qualificadas, em particular no ambiente internacional, a considerar a base de dados JCR/ISI (Journal Citations Report) como índice Fator de Impacto na avaliação dos periódicos.

$\mathrm{O}$ total de artigos pesquisados pode ser verificado na Tabela 2 , a seguir.

Tabela 2 - Total de Artigos pesquisados

\begin{tabular}{c|c|c}
\hline Tipo & Total & $\%$ \\
\hline A2 & 1.837 & $13 \%$ \\
\hline B1 & 4.868 & $35 \%$ \\
\hline B2 & 2.014 & $14 \%$ \\
\hline B3 & 5.216 & $37 \%$ \\
\hline TOTAL & $\mathbf{1 3 . 9 3 5}$ & $\mathbf{1 0 0 \%}$ \\
\hline
\end{tabular}

Fonte: Dados dos autores, 2012.

Foram pesquisados 13.935 periódicos na busca por artigos nacionais de Auditoria Contínua. Após análise da Tabela 2, verifica-se que o periódico do tipo B3 apresenta um percentual maior, em sequência, o tipo B1 e bem próximos o B4 e B3 respectivamente por maior concentração de publicações.

Já na Tabela 3, é apresentada uma análise comparativa do número de publicações diversas com os artigos de Auditoria e Auditoria Contínua devidamente evidenciados e excluídos do cálculo total de publicações por cada tipo de avaliação Capes.

Tabela 3 - Total Comparativo de Artigos de Auditoria Publicados em Periódicos Brasileiros entre 2006 e 2011

\begin{tabular}{c|c|c|c|c|c}
\hline Tipo & $\begin{array}{c}\text { Publicações } \\
\text { Diversas }\end{array}$ & Auditoria & $\begin{array}{c}\text { Percentual de } \\
\text { Auditoria }\end{array}$ & $\begin{array}{c}\text { Auditoria } \\
\text { Contínua }\end{array}$ & $\begin{array}{c}\text { Percentual } \\
\text { Auditoria } \\
\text { Contínua }\end{array}$ \\
\hline A2 & 1.820 & 17 & $0,93 \%$ & - & - \\
\hline B1 & 4.844 & 24 & $0,50 \%$ & 3 & $0,06 \%$ \\
\hline B2 & 2.005 & 9 & $0,45 \%$ & - & - \\
\hline B3 & 5.191 & 25 & $0,48 \%$ & - & - \\
\hline TOTAL & $\mathbf{1 3 . 8 6 0}$ & $\mathbf{7 5}$ & & $\mathbf{3}$ & \\
\hline
\end{tabular}

Fonte: Dados dos autores, 2012.

A tabela 3 faz a distinção de publicações diversas, ou seja, artigos publicados com temas variados de acordo com a proposta do periódico das áreas de concentração pesquisadas conforme o quadro 2, com os artigos de Auditoria e Auditoria Contínua. Observa-se que 
embora o tipo B3 e B1, respectivamente, indicam uma maior publicação abordando Auditoria, estes índices não ultrapassam a $1 \%$ do total de publicações do mesmo tipo de periódico. Portanto, há evidencias de carência com os estudos científicos sobre esse assunto. Tão pouco a Auditoria Contínua, que só teve apenas 3 publicações em um único tipo de periódico: B1, o que em termos percentuais representa $0,06 \%$.

Pela Tabela 4 é possível acompanhar a evolução dos artigos de auditoria entre os anos de 2006 e 2011.

Tabela 4 - Evolução dos Artigos sobre Auditoria em Periódicos entre 2006 e 2011

\begin{tabular}{c|c|c|c|c|c|c}
\hline Tipo & $\mathbf{2 0 0 6}$ & $\mathbf{2 0 0 7}$ & $\mathbf{2 0 0 8}$ & $\mathbf{2 0 0 9}$ & $\mathbf{2 0 1 0}$ & $\mathbf{2 0 1 1}$ \\
\hline A2 & 4 & 3 & 4 & 3 & 1 & 2 \\
\hline B1 & 2 & 1 & 4 & 6 & 6 & 5 \\
\hline B2 & 1 & 2 & 4 & 0 & 1 & 1 \\
\hline B3 & 2 & 5 & 5 & 3 & 10 & 0 \\
\hline TOTAL & $\mathbf{9}$ & $\mathbf{1 1}$ & $\mathbf{1 7}$ & $\mathbf{1 2}$ & $\mathbf{1 8}$ & $\mathbf{8}$ \\
\hline
\end{tabular}

Fonte: Dados dos autores, 2012.

A tabela 4 lista o total dos artigos sobre Auditoria, sendo possível acompanhar a sua evolução quantitativa no decorrer de 2006 até 2011. Nota-se que os anos de 2008 e 2010 apresentaram, respectivamente, os maiores índices com o total de 17 e 18 publicações. No entanto, percebese que nos anos de 2009 e 2011, ocorreu redução nas publicações. Logo, conclui-se que não existe ritmo padrão de crescimento, redução ou uniformidade entre os períodos analisados. A Tabela 5 destaca os artigos brasileiros sobre a Auditoria Contínua entre 2006 e 2011.

Tabela 5 - Artigos sobre Auditoria Contínua publicados entre 2006 e 2011

\begin{tabular}{c|c|c|c|c|c|c}
\hline Tipo & $\mathbf{2 0 0 6}$ & $\mathbf{2 0 0 7}$ & $\mathbf{2 0 0 8}$ & $\mathbf{2 0 0 9}$ & $\mathbf{2 0 1 0}$ & $\mathbf{2 0 1 1}$ \\
\hline A2 & 0 & 0 & 0 & 0 & 0 & 0 \\
\hline B1 & 2 & 0 & 0 & 0 & 1 & 0 \\
\hline B2 & 0 & 0 & 0 & 0 & 0 & 0 \\
\hline B3 & 0 & 0 & 0 & 0 & 0 & 0 \\
\hline TOTAL & $\mathbf{2}$ & $\mathbf{0}$ & $\mathbf{0}$ & $\mathbf{0}$ & $\mathbf{1}$ & $\mathbf{0}$ \\
\hline
\end{tabular}

Fonte: Dados dos autores, 2012.

Pela análise da Tabela 5 pode-se constatar que a Auditoria Contínua não é um assunto explorado no contexto brasileiro. Foram identificadas apenas divulgação no periódico B1, tendo 2 publicações em 2006 e 1 publicação em 2010. Entre os anos de 2006 e 2011 destacase a referência destas publicações em um único periódico, a revista de Gestão de Tecnologia e Sistemas de Informação da Universidade de São Paulo (USP).

\section{CONSIDERAÇÕES FINAIS}

O presente estudo teve como objetivo abordar as tendências de pesquisa sobre a Auditoria Contínua em trabalhos publicados em periódicos brasileiros A2, B1, B2 e B3 no período de 2006 a 2011. Para o desenvolvimento deste estudo, foram pesquisados os conceitos desenvolvidos na Auditoria Contínua, a metodologia aplicada, a consulta e a referência da bibliografia utilizada.

Observa-se que a adoção da Auditoria Contínua pelas instituições pode refletir tanto uma obrigatoriedade legal da expansão das responsabilidades dos Administradores, no que diz respeito à eficiência e eficácias dos controles internos e relatórios financeiros, como também, uma estratégia de negócios, pois produz informações instantâneas de qualidade e confiáveis 
para a tomada de decisões não apenas gerenciais, mas também de interesse aos usuários de uma forma geral (investidores, fornecedores, governo e sociedade).

Os resultados das pesquisas apresentados nos periódicos internacionais analisados neste artigo (Quadro 1) são favoráveis ao uso da Auditoria Contínua, apesar de considerar as limitações pertinentes ao desenvolvimento do tema. São propostas de novas abordagens sobre o tema, como a ampliação dos testes de hipóteses realizados, pois foi constatado nos referidos artigos que a adoção da Auditoria Contínua representa uma tendência das grandes empresas nos setores de telecomunicações e bancário.

Embora, o contexto brasileiro apresente apenas duas publicações, em 2006 e uma publicação, em 2010, sobre a Auditoria Contínua na revista de Gestão de Tecnologia e Sistemas de Informação pela USP, os estudos abordados identificam uma tendência natural no uso da Auditoria Contínua no Brasil para acompanhar o nível de exigência jurídica dos órgãos reguladores, relativos à necessidade de maior rapidez, precisão e confiabilidade no acesso aos relatórios de auditoria.

No aspecto jurídico, foi constatado, com o artigo publicado em 2010, que trata sobre o $20^{\circ}$ Simpósio Mundial sobre Auditoria Contínua, o interesse de órgãos de controle, como o Tribunal de Contas da União, na participação de debates sobre Auditoria Contínua, mostrando um indicativo de que o governo brasileiro pode estar indicando uma revisão de suas leis e preparando um campo propício para o início da implantação da Auditoria Contínua no Brasil.

As pesquisas indicam que a aplicação da Auditoria Contínua não contempla somente o avanço tecnológico, mas, também, o nível de capacitação profissional exigido do auditor, fundamental, para identificar, avaliar e gerenciar as informações geradas no ambiente computadorizado, bem como, a estrutura organizacional inserida no contexto e os agentes externos de regulamentação dos mercados que interferem tanto na sua implementação quanto na sua continuidade.

Portanto, faz-se necessário o incentivo para a realização de pesquisas nacionais, para que Brasil venha a contribuir com estudos na área de Auditoria Contínua na avaliação de sua adequação e aplicabilidade à sua realidade cultural, social e econômica.

Cabe ressaltar que as evidências, achados e conclusões, não devem ser generalizadas, uma vez que, existem limitações temporais e metodológicas do universo pesquisado. Desta maneira, o presente estudo pode ser entendido como tendência indicativa da evolução do tema em estudo, em um cenário caracterizado como a Sociedade da Informação.

\section{REFERÊNCIAS}

ALLES, M.G.; KOGAN, A.; VASARHELYI, M.A. A Lei das Consequências não Intencionais? Avaliando os Custos, Benefícios e Resultados da Lei Sarbanes-Oxley. São Paulo: ISACA, 2004. Disponível em: <http://www.isaca.org.br/publicacoes/a_lei_das_ consequencias_nao_intencionais_sarbanes_oxley.pdf >. Acesso em: 1 abr. 2012.

ALLES, M.G.; KOGAN, A.; VASARHELYI, M.A. Feasibility and Economics of Continuous Assurance. Auditing: A Journal of Practice and Theory, v. 21, n. 1. 2002.

ALLES, M. G.; TOSTES, F. P.; VASARHELYI, M. A.; RICCIO, E. L. Continuous Auditing: The USA Experience and Considerations for its Implementation in Brazil. Revista de Gestão da Tecnologia e Sistemas de Informação. Journal of Information Systems and Technology Management. V. 3, n. 2. 2006

CERVO, A. L.; BERVIAN, P. A. Metodologia Científica. 3 ed. São Paulo: McGraw-Hill do Brasil, 1983. 
CHANG, S.; WU, C. C.; CHANG, I. The Development of a Computer Auditing System Sufficient for Sarbanes-Oxley Section 404 - A Study on the Purchasing and Expenditure Cycle of the ERP System. Information Systems Management, v. 25, 2008.

CHOU, C. C.; CHANG, C. J. Continuous auditing for web-released financial information, Review of Accounting and Finance, v. 9, n. 1. 2010.

CAPES - Coordenação de Aperfeiçoamento de Pessoal de Nível Superior. Disponível em $<$ htttp://www.capes.gov.br>. Acesso em: 2 jun. 2012.

COLLIS, J.; HUSSEY R. Pesquisa em Administração: um guia prático para alunos de graduação e pós-graduação, 2 ed. Porto Alegre, Bookman, 2005.

EL-MASRY, E. E; RECK, J. L. Continuous online auditing as a response to the SarbanesOxley Act, Managerial Auditing Journal, v. 23, n. 8. 2008.

FORMIGA, A. L.; ALVES, C. R. G.; MARQUES, E. P. P. Auditoria Integral. Trabalho de Pós-Graduação, Faculdade Bom Jesus, FAE Business School. Curitiba, 2004.

GROOMER, M.; MURTHY, U. Continuous auditing of database applications: An embedded audit module approach. Journal of Information Systems, Spring, v. 3, n. 2. 1989.

KANELLOU, A.; SPATHIS, C. Auditing in enterprise system environment: a synthesis. Journal of Enterprise Information Management, v. 24, n. 6. 2011.

KOGAN, A.; SUDIT, E.F.; VASARHELYI, M.A. Continuous Online Auditing: A Program of Research, Journal of Information Systems, v. 1, n. 3. 1999.

KOSKIVAARA, E. Integrating Analytical Procedures into the Continuous Audit Environment, Journal of Information Systems and Technology Management, v. 3, n. 3, 2007.

MARION, J. C. Preparando-se para a profissão do futuro. Revista Razão Contábil. Maio, 2003.

NASI, A. C. A auditoria integral como instrumento de uma gestão eficaz e voltada para novas exigências dos usuários: um desafio definitivo para o auditor independente como agente do desenvolvimento empresarial. Trabalho apresentado na XXII Conferência Interamericana de Contabilidade, 1997.

OMOTESO K.; PATEL A.; SCOTT P. An Investigation into the Application of Continuous Online Auditing in the U.K.. The International Journal of Digital Accounting Research. v. 8, n. 14, 2008.

REZAEE, Z.; SHARBATOGHLIE, A.; ELAM, R.; MCMICKLE, P. L. Continuous auditing: building automated auditing capability. Auditing: A Journal of Practice \& Theory. v. 21. 2002.

RICCIO, E. L.; SAKATA, M. C. G.; VALENTE, N. T. Z. Resultados do $7^{\circ}$ CONTECSI Congresso Internacional de Gestão da Tecnologia e Sistemas de Informação. Journal of Information Systems and Technology Management, v. 7, n. 2. 2010. 
SEARCY, D.; WOODROOF J.; BRUCE, B. Continuous Audit: The Motivations Benefits, Problems, and Challenges Identified by Partners of a Big 4 Accounting Firm.

Proceedings of the 36th Hawaii International Conference on System Sciences, 2002.

THOMAS, M. A.; MARATHE, R. R. Determining expected behavior of fraudsters for a continuous audit system. IIMB Management Review. 2012.

TOSTES, F. P. Gestão de risco de mercado: metodologias financeira e contábil. FGV Editora. 1 ed., 2007.

AQUINO, C.E.M.; SILVA, W. L.; SIGOLO, N.; VASARHELYI, M.A. Six Steps to an Effective Continuous Audit Process. The Tech Forum, Institute of Internal Auditors. Julho, 2008.

VOSS, J. A Auditoria Integral: Um Pulo no Vazio. Boletim do IBRACON, ANO XXI, p.315, 1999. 